\title{
Corticospinal tract atrophy and motor fMRI predict motor preservation after functional cerebral hemispherectomy
}

\author{
Anthony C. Wang, MD, ${ }^{1}$ George M. Ibrahim, MD, PhD, ${ }^{3}$ Andrew V. Poliakov, PhD, ${ }^{4}$ \\ Page I. Wang, MD, ${ }^{1}$ Aria Fallah, MD, MSc, ${ }^{1}$ Gary W. Mathern, MD, ${ }^{1,2}$ Robert T. Buckley, MD, ${ }^{4}$ \\ Kelly Collins, MD, ${ }^{4}$ Alexander G. Weil, MD, ${ }^{7}$ Hillary A. Shurtleff, PhD, ${ }^{5}$ Molly H. Warner, PhD, ${ }^{5}$ \\ Francisco A. Perez, MD, PhD, ${ }^{6}$ Dennis W. Shaw, MD, ${ }^{6}$ Jason N. Wright, MD, ${ }^{6}$ \\ Russell P. Saneto, DO, PhD, ${ }^{5}$ Edward J. Novotny, MD, ${ }^{5}$ Amy Lee, MD, ${ }^{4}$ Samuel R. Browd, MD, PhD, ${ }^{4}$ \\ and Jeffrey G. Ojemann, MD ${ }^{4}$
}

Departments of ${ }^{1}$ Neurosurgery and ${ }^{2}$ Psychiatry and BioBehavioral Sciences, The Brain Research Institute, University of California, Los Angeles, California; ${ }^{3}$ Division of Neurosurgery, Hospital for Sick Children and Toronto Western Hospital, Toronto, Ontario, Canada; Departments of ${ }^{4}$ Neurological Surgery, ${ }^{5}$ Neurology/Division of Pediatric Neurology, and ${ }^{6}$ Radiology, University of Washington, Seattle Children's Hospital, Seattle, Washington; and 'Division of Pediatric Neurosurgery, Department of Surgery, Sainte Justine Hospital, University of Montreal, Quebec, Canada

OBJECTIVE The potential loss of motor function after cerebral hemispherectomy is a common cause of anguish for patients, their families, and their physicians. The deficits these patients face are individually unique, but as a whole they provide a framework to understand the mechanisms underlying cortical reorganization of motor function. This study investigated whether preoperative functional MRI (fMRI) and diffusion tensor imaging (DTI) could predict the postoperative preservation of hand motor function.

METHODS Thirteen independent reviewers analyzed sensorimotor fMRI and colored fractional anisotropy (CoFA)-DTI maps in 25 patients undergoing functional hemispherectomy for treatment of intractable seizures. Pre- and postoperative gross hand motor function were categorized and correlated with fMRI and DTI findings, specifically, abnormally located motor activation on fMRI and corticospinal tract atrophy on DTI.

RESULTS Normal sensorimotor cortical activation on preoperative fMRI was significantly associated with severe decline in postoperative motor function, demonstrating $92.9 \%$ sensitivity $(95 \% \mathrm{Cl} 0.661-0.998)$ and $100 \%$ specificity $(95 \%$ $\mathrm{Cl} 0.715-1.00)$. Bilaterally robust, symmetric corticospinal tracts on CoFA-DTI maps were significantly associated with severe postoperative motor decline, demonstrating $85.7 \%$ sensitivity $(95 \% \mathrm{Cl} 0.572-0.982)$ and $100 \%$ specificity $(95 \%$ $\mathrm{Cl} 0.715-1.00)$. Interpreting the fMR images, the reviewers achieved a Fleiss' kappa coefficient ( $\kappa)$ for interrater agreement of $\kappa=0.69$, indicating good agreement $(p<0.01)$. When interpreting the CoFA-DTI maps, the reviewers achieved $\kappa$ $=0.64$, again indicating good agreement $(p<0.01)$.

CONCLUSIONS Functional hemispherectomy offers a high potential for seizure freedom without debilitating functional deficits in certain instances. Patients likely to retain preoperative motor function can be identified prior to hemispherectomy, where fMRI or DTI suggests that cortical reorganization of motor function has occurred prior to the operation.

https://thejns.org/doi/abs/10.3171/2017.7.PEDS17137

KEY WORDS hemispherectomy; reorganization; functional MRI; fMRI; diffusion tensor imaging; DTI; epilepsy

I NDIVIDUALS who have suffered extensive brain injury in utero or during the perinatal period can demonstrate intellectual and physical abilities that seem exceptional, given the extent of encephalomalacia and other signs of brain damage noted on neuroimaging studies. A propor- tion of these patients also develop seizure activity that can be difficult to control with antiepileptic medications alone and may be candidates for cerebral hemispherectomy, which can offer a chance of seizure freedom as high as $80 \% .^{7,16,18,22}$

ABBREVIATIONS COFA = colored fractional anisotropy; DTI = diffusion tensor imaging; $f M R I=$ functional MRI; SMA = supplementary motor area .

SUBMITTED March 16, 2017. ACCEPTED July 12, 2017.

INCLUDE WHEN CITING Published online November 3, 2017; DOI: 10.3171/2017.7.PEDS17137. 
An estimated $18 \%-36.5 \%$ of patients undergoing functional hemispherectomy witness no decrement in their baseline motor function after surgical intervention. ${ }^{8,23}$ This observation suggests that motor pathway reorganization and/or disinhibition occurred either prior to surgery or alternative pathways were unmasked as a result of surgical intervention. Several investigators have attempted to identify the cortical and subcortical regions associated with the neuroplasticity of motor function in patients who have undergone functional hemispherectomy. ${ }^{1,20,27-30}$ In addition to the primary motor cortex, associative cortical areasincluding the supplementary motor area (SMA), insula, inferior frontal cortex, occipital cortex, basal ganglia, and cerebellum-have been investigated as potential contributors to motor function preservation.

Utilization and disinhibition of the ipsilateral motor pathways are commonly thought to be a primary mechanism by which functional preservation occurs for several reasons. First, robust ipsilateral pathways are present at birth, representing an estimated $25 \%$ of descending uncrossed corticospinal fibers that contribute to the lateral and ventral corticospinal tracts. ${ }^{1}$ Demand may then lead to increased ipsilateral tract utilization. Likewise, crossed inhibitory signaling from the contralateral hemisphere may lead to inhibition of the ipsilateral tracts in the preoperative state. Release from contralateral inhibition via hemispherectomy might thus facilitate ipsilateral corticospinal control of limb function. ${ }^{13}$ Disinhibition has also been proposed as a mechanism for the mirror movements commonly seen in patients who have undergone hemispherectomy, while crowding of motor function on the remaining motor cortex is another leading theory.

The location of reorganization within the remaining unaffected hemisphere has been studied and debated. Reorganization has been observed in the peri-rolandic cortex, both medial and lateral to motor activation of the nonparetic limb. ${ }^{14}$ Restoration or preservation of function is also hypothesized to be supported by areas outside of the primary motor cortex, including the premotor, SMA, and rubrospinal pathways. ${ }^{28}$ Staudt et al. argued that reorganization is dependent on the extent of the lesion, with severe lesions leading to disruption of interhemispheric inhibitory connections via the corpus callosum, including crossed descending corticospinal tracts. ${ }^{35}$

Few researchers have used advanced imaging techniques such as functional MRI (fMRI) or diffusion tensor imaging (DTI) to investigate the preservation of motor function relative to the severe hemiparesis expected in patients about to undergo hemispherectomy., ${ }^{4,519,24,26,39}$ In this observational study, we evaluate the laterality of hand motor control using fMRI, as well as corticospinal tract integrity via colored fractional anisotropy $(\mathrm{CoFA})$ maps, in patients undergoing functional hemispherectomy for medically intractable seizures (Fig. 1). The goal of our study was to determine whether these imaging techniques can be used to identify the patients most likely to retain motor function postoperatively.

\section{Methods}

Institutional review board approval was obtained to waive informed consent prior to performing a retrospective review of clinical and imaging data collected from 25 patients who underwent functional hemispherectomy to treat medically intractable seizures from 2007 to 2015 at our institution. The technique used most closely resembles "peri-insular hemispherotomy" as described by Schramm et al., ${ }^{33}$ while typically resecting the insula and basal forebrain and sparing the caudate nucleus and thalamus. Each patient underwent fMRI in the months preceding surgery as part of the preoperative workup. DTI was performed as part of preoperative structural MRI. The full imaging studies were interpreted by fellowship-trained pediatric neuroradiologists and reviewed by fellowship-trained pediatric neurosurgeons. Culled from patient records, immediate preoperative motor strength was compared against the last known postoperative motor strength testing and classified as mildly or severely weaker or stable.

\section{Motor fMRI Acquisition}

Imaging was performed on a Siemens 3-T Trio scanner. fMRI was performed using EPIBOLD images (Siemens clinical sequence ep2d_pace 45 slices; 3-mm-thick slices with a $0-\mathrm{mm}$ gap; TR/TE $=2420 / 30 \mathrm{msec}$; flip angle $90^{\circ}$; field of view $192 \times 192 \mathrm{~mm}$; matrix $64 \times 64$ ). A total of 80 image volumes were obtained per run, resulting in a total time of less than 7 minutes per run. Standard DTI parameters were used: TR $5800 \mathrm{msec}$, TE $96 \mathrm{msec} ; \mathrm{b}=$ $1000 \mathrm{sec} / \mathrm{mm}^{2} ; 10-30$ diffusion directions repeated 2-4 times; in-plane resolution $1.8 \times 1.8 \mathrm{~mm}$; and $3-\mathrm{mm}$ slice thickness.

Motor fMRI tasks were performed according to our previously published parameters. ${ }^{34}$ Motor tasks alternated between left- and right-hand movements (visually cued, self-paced, tapping of the thumb and fingers together). Studies used a conventional block design (ABABAB paradigm) and were repeated at least twice. When the patient could not voluntarily perform the tapping on the affected side, the task was aided by a parent who passively moved the relevant digits as the patient participated at the appropriate times and kept the unattended hand still.

\section{Postprocessing and Image Analysis}

Analysis of the fMRI and DTI data was performed using commercial Siemens software, and images were also analyzed using the FSL/FDT software package (FMRIB Image Analysis Group; www.fmrib.ox.ac.uk/fsl). fMRI data processing was carried out using FEAT (FMRI Expert Analysis Tool; version 5.92). The following prestatistical processing was applied: motion correction using MCFLIRT; nonbrain removal using BET; spatial smoothing using a Gaussian kernel with a 5-mm full width at half maximum; grand mean intensity normalization of the entire 4-dimensional data set by a single multiplicative factor; high-pass temporal filtering (Gaussian-weighted least-squares straight line fitting with $\sigma=50.0$ seconds). Statistical analysis of the time series was carried out using FILM with local autocorrelation correction. Multiple trials were aggregated for cluster enhancement with $\mathrm{Z}$ (Gaussianized T/F) statistic image threshold $>3.0$ and a corrected cluster significance threshold of $p=0.01$. Regis- 

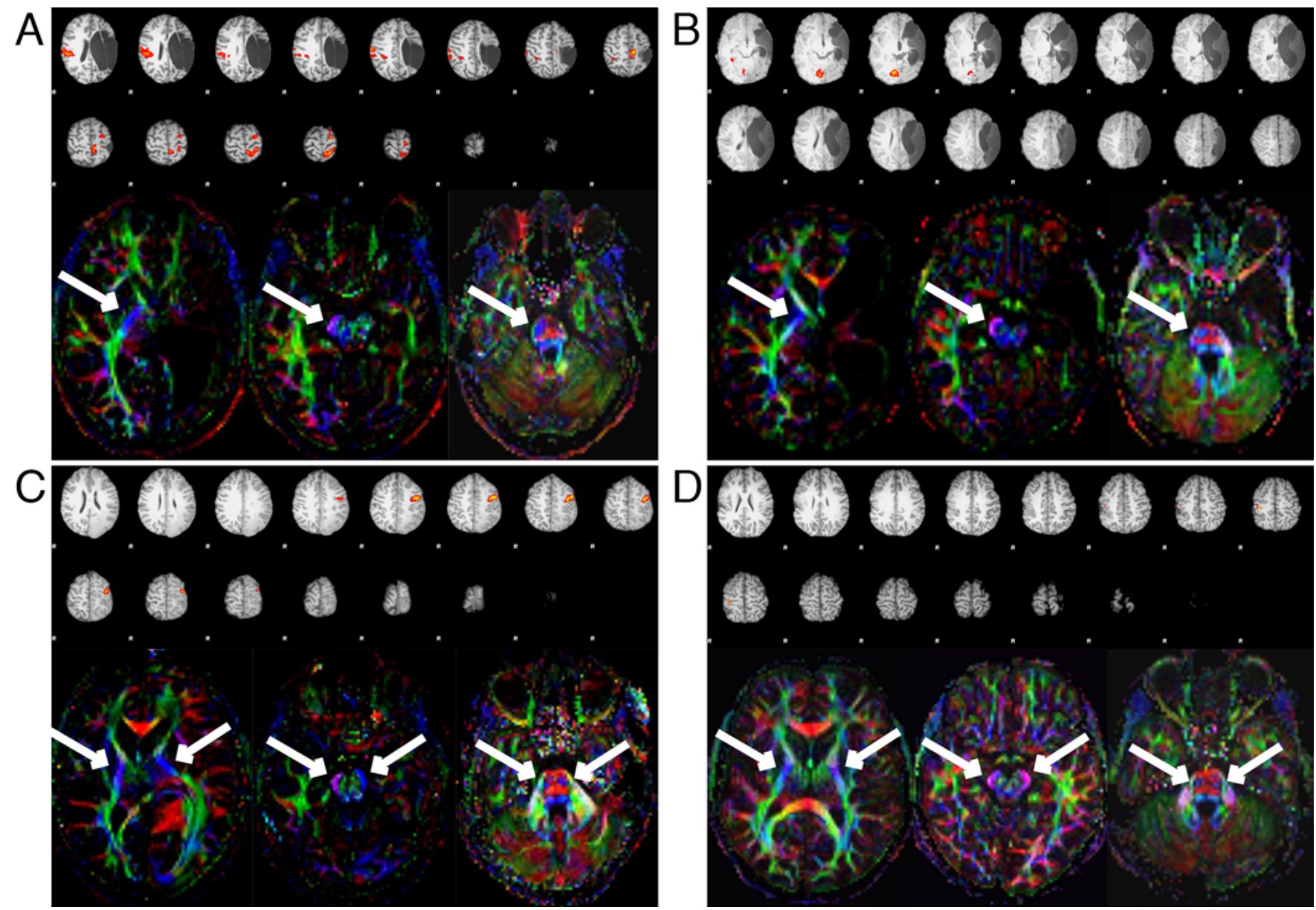

FIG. 1. Representative CoFA and fMRI images. A and B: Representative CoFA-DTI images demonstrating minimal corticospinal tracts (arrows) extending from the damaged hemisphere in 2 different patients, with apparent motor reorganization on preoperative fMRI. DTI emphasizes the near absence of the corticospinal motor tracts at the posterior limb of the internal capsule, cerebral peduncle, and upper medulla. Neither of these patients experienced new neurological deficit after hemispherectomy. C and D: In contrast, CoFA-DTI images from 2 patients with normal contralateral motor fMRI results show the essentially symmetric bulk of the corticospinal tracts (arrows) at the internal capsule, cerebral peduncle, and upper medulla. Both of these patients experienced severe contralateral hemiparesis after hemispherectomy.

tration to high-resolution structural statistical parametric maps was carried out using FLIRT.

Analysis of the DTI data included eddy current correction, fitting of diffusion tensors, and estimation of diffusion parameters including fractional anisotropy, principal diffusion direction, and mean diffusivity. Evaluation of the DTI data was done by examining quantitative parametric maps and the direction-encoded CoFA maps. Fiber tracking algorithms were performed using both the FSL and MedINRIA software packages (Asclepios Research Project; http://www-sop.inria.fr/asclepios/software/ MedINRIA/). The corticospinal tract within the posterior limbs of the internal capsule, cerebral peduncle, and upper medulla was compared with that of the contralateral uninjured side.

\section{Interrater Reliability}

The resulting statistical parametric maps generated using fMRI and CoFA-DTI data were visually rated by 13 independent reviewers (7 neurosurgeons, 2 neurologists, and 4 radiologists) using categorical scales. For fMRI data, raters were asked to determine whether sensorimotor activation was absent, ipsilateral sensorimotor adjacent, or contralateral to the paretic limb. For the DTI data, raters scored the CoFA maps as symmetric and robust on both sides or significantly diminished on 1 side. The images used for evaluation are available in Appendix A. For each individual case, the majority interpretation of that case was used for analysis. To index interrater reliability, Fleiss' kappa coefficient (К) was calculated. ${ }^{9}$ Analysis was performed using R statistical software and the "irr" package (version 0.84).

\section{Results}

\section{Patient Population}

Of the 25 patients with functional hemispherectomy patients who were reviewed, 11 patients were male and 14 patients were female. The mean age at seizure onset was 3.16 years (SD 4.11 years), and the mean age at the 
TABLE 1. Individual patient data

\begin{tabular}{|c|c|c|c|c|c|c|c|c|c|}
\hline \multirow[b]{2}{*}{$\begin{array}{l}\text { Case } \\
\text { No. }\end{array}$} & \multirow[b]{2}{*}{ Sex } & \multirow{2}{*}{$\begin{array}{l}\text { Age at } \\
\text { Seizure } \\
\text { Onset } \\
\text { (yrs) }\end{array}$} & \multirow[b]{2}{*}{ Etiology } & \multirow[b]{2}{*}{$\begin{array}{c}\text { Side of } \\
\text { Hemispherectomy }\end{array}$} & \multirow{2}{*}{$\begin{array}{c}\text { Age at } \\
\text { Hemispherectomy } \\
\text { (yrs) }\end{array}$} & \multicolumn{2}{|c|}{ Motor Function } & \multirow{2}{*}{$\begin{array}{l}\text { fMRI }^{*} \\
\text { Preop }\end{array}$} & \multirow{2}{*}{$\begin{array}{l}\text { DTI† } \\
\text { Preop }\end{array}$} \\
\hline & & & & & & Preop & Postop & & \\
\hline 1 & M & 0.0 & Perinatal stroke w/ ICH & Rt & 18.2 & Mild It hemiparesis & Severely weaker & Contralat & Symmetric \\
\hline 2 & M & 1.0 & Perinatal stroke & $\mathrm{Lt}$ & 16.8 & Mod rt hemiparesis & Stable & Ipsilat & Atrophic \\
\hline 3 & $\mathrm{~F}$ & 4.2 & Perinatal stroke & $\mathrm{Lt}$ & 16.8 & Mod rt hemiparesis & Stable & Ipsilat & Atrophic \\
\hline 4 & $\mathrm{~F}$ & 4.8 & Perinatal stroke w/ ICH & $\mathrm{Lt}$ & 10.1 & Mod rt hemiparesis & Stable & Absent & Atrophic \\
\hline 5 & $\mathrm{~F}$ & 6.6 & Perinatal stroke & Rt & 7.9 & Mod It hemiparesis & Mildly weaker & Absent & Atrophic \\
\hline 6 & $\mathrm{~F}$ & 1.1 & Perinatal stroke & Rt & 6.7 & Mild It hemiparesis & Severely weaker & Contralat & Symmetric \\
\hline 7 & $\mathrm{~F}$ & 0.0 & Perinatal stroke & $\mathrm{Lt}$ & 6.0 & $\begin{array}{l}\text { Severe rt } \\
\text { hemiparesis }\end{array}$ & Stable & Ipsilat & Atrophic \\
\hline 8 & M & 2.7 & Perinatal stroke & $\mathrm{Lt}$ & 5.9 & Mod rt hemiparesis & Severely weaker & Contralat & Atrophic \\
\hline 9 & $M$ & 0.9 & Perinatal stroke & $\mathrm{Lt}$ & 5.7 & Mild rt hemiparesis & Stable & Ipsilat & Atrophic \\
\hline 10 & $M$ & 0.6 & Perinatal stroke & Rt & 2.5 & Mod It hemiparesis & Stable & Absent & Atrophic \\
\hline 11 & $M$ & 0.0 & Perinatal stroke & $\mathrm{Lt}$ & 1.3 & Mod rt hemiparesis & Mildly weaker & Ipsilat & Atrophic \\
\hline 12 & $\mathrm{~F}$ & 10.2 & Childhood stroke & Rt & 13.1 & Mod It hemiparesis & Severely weaker & Contralat & Symmetric \\
\hline 13 & $\mathrm{M}$ & 16.0 & Hemimegalencephaly & $\mathrm{Lt}$ & 17.1 & Mild rt hemiparesis & Severely weaker & Contralat & Symmetric \\
\hline 14 & $F$ & 0.1 & Hemimegalencephaly & $\mathrm{Rt}$ & 6.1 & Mod It hemiparesis & Stable & Absent & Atrophic \\
\hline 15 & $\mathrm{~F}$ & 0.0 & Hemimegalencephaly & $\mathrm{Lt}$ & 1.0 & $\begin{array}{l}\text { Minimal rt } \\
\text { hemiparesis }\end{array}$ & Severely weaker & Contralat & Symmetric \\
\hline 16 & M & 0.4 & Cortical dysplasia & $\mathrm{Lt}$ & 2.1 & $\begin{array}{l}\text { Minimal rt } \\
\text { hemiparesis }\end{array}$ & Severely weaker & Absent & Symmetric \\
\hline 17 & $\mathrm{~F}$ & 0.1 & Cortical dysplasia & $\mathrm{Lt}$ & 1.1 & Mild rt hemiparesis & Severely weaker & Contralat & Symmetric \\
\hline 18 & $F$ & 0.0 & Cortical dysplasia & $\mathrm{Lt}$ & 12.7 & $\begin{array}{l}\text { Minimal rt } \\
\text { hemiparesis }\end{array}$ & Severely weaker & Contralat & Symmetric \\
\hline 19 & $\mathrm{~F}$ & 0.1 & $\begin{array}{l}\text { Rasmussen } \\
\text { encephalitis }\end{array}$ & $\mathrm{Rt}$ & 2.7 & $\begin{array}{l}\text { Minimal It } \\
\text { hemiparesis }\end{array}$ & Severely weaker & Contralat & Symmetric \\
\hline 20 & M & 5.0 & $\begin{array}{l}\text { Rasmussen } \\
\text { encephalitis }\end{array}$ & $\mathrm{Rt}$ & 9.7 & Mod It hemiparesis & Severely weaker & Contralat & Symmetric \\
\hline 21 & $\mathrm{~F}$ & 1.2 & Sturge-Weber & $\mathrm{Lt}$ & 3.1 & Mod rt hemiparesis & Severely weaker & Contralat & Symmetric \\
\hline 22 & $\mathrm{M}$ & 4.0 & Polymicrogyria & Rt & 11.4 & Mild It hemiparesis & Severely weaker & Contralat & Symmetric \\
\hline 23 & $F$ & 4.0 & Polymicrogyria & $\mathrm{Lt}$ & 11.7 & $\begin{array}{l}\text { Severe rt } \\
\text { hemiparesis }\end{array}$ & Stable & Ipsilat & Atrophic \\
\hline 24 & $M$ & 10.7 & Glioblastoma & $\mathrm{Rt}$ & 12.4 & Mod hemiparesis & Severely weaker & Contralat & Atrophic \\
\hline 25 & $F$ & 5.2 & Low-grade glioma & $\mathrm{Lt}$ & 6.9 & Mod rt hemiparesis & Stable & Absent & Atrophic \\
\hline
\end{tabular}

$\mathrm{ICH}=$ intracerebral hemorrhage; mod $=$ moderate.

* The primary site of $\mathrm{MMRI}$ activation is given relative to the affected limbs.

† The integrity of the corticospinal tract was assessed at the posterior limbs of the internal capsule, midbrain, and medulla.

time of hemispherectomy was 8.36 years (SD 5.47 years). Ten operations were performed on the right side and 15 on the left side. Eleven patients developed seizures related to perinatal stroke; 2 of these patients also had experienced intracerebral hemorrhage. One patient had a stroke at the age of 10 years, and seizures began shortly thereafter. Three patients had hemimegalencephaly, 3 patients had cortical dysplasia, 2 patients had Rasmussen encephalitis, 2 patients had polymicrogyria, 1 patient had Sturge-Weber syndrome, 1 patient had a high-grade glioma, and 1 patient had a low-grade glioma.

\section{Summary of Findings}

Preoperative and postoperative motor function, preop- erative findings of motor activation on brain $\mathrm{fMRI}$ relative to finger movements on the injured side, and qualitative evaluation of the corticospinal tracts on the preoperative CoFA-DTI maps for each patient are listed in Table 1. These results are represented in Fig. 2, which compares the imaging results with motor outcomes.

On preoperative fMRI, 6 of 25 patients (24.0\%) showed activation in the sensorimotor-adjacent regions ipsilateral to the paretic hand, 6 patients $(24.0 \%)$ showed no interpretable activation, and 13 patients $(52.0 \%)$ showed normal contralateral sensorimotor region activation. Of the 6 patients showing ipsilateral activity, 5 patients $(83.3 \%)$ remained stable and 1 patient $(16.7 \%)$ was subtly weaker postoperatively. No patients experienced severe postoper- 


\begin{tabular}{lccc}
\hline fMR & Stable or improved & Mildly weaker & Severely weaker \\
\hline All patients & & & \\
\hline Ipsilateral & 5 & 1 & 0 \\
\hline None & 4 & 1 & 1 \\
\hline Contralateral & 0 & 0 & 13 \\
\hline Stroke patients & & & \\
\hline Ipsilateral & 4 & 1 & 0 \\
\hline None & 2 & 1 & 0 \\
\hline Contralateral & 0 & 0 & 3 \\
\hline
\end{tabular}

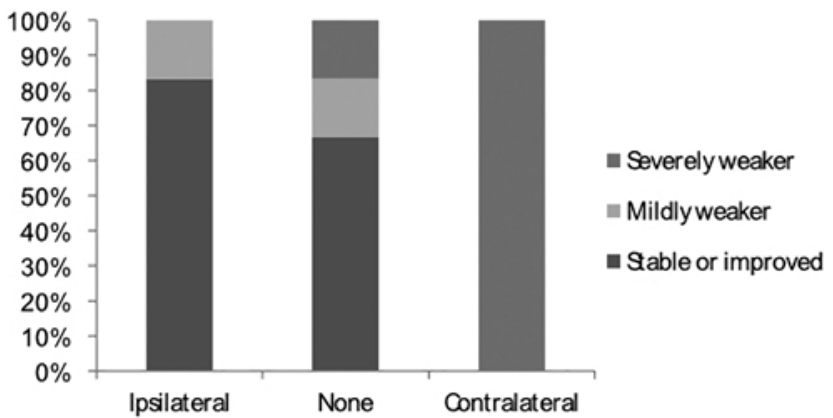

\begin{tabular}{|cccc}
\hline DT & Stable or improved & Mildly weaker & Severely weaker \\
\hline All patients & & & \\
\hline Diminished & 9 & 2 & 2 \\
\hline Symmetric & 0 & 0 & 12 \\
\hline Stroke patients & & & \\
\hline Diminished & 6 & 2 & 1 \\
\hline Symmetric & 0 & 0 & 2 \\
\hline
\end{tabular}

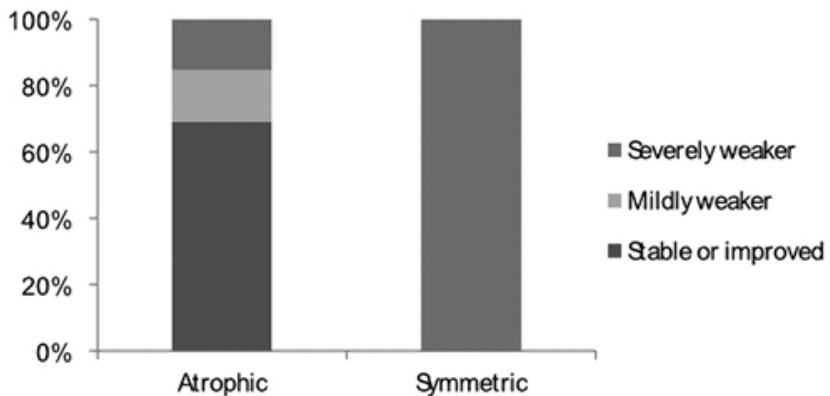

FIG. 2. Comparisons of the imaging and motor results. The Freeman-Halton extension of the Fisher's exact test was performed on the contingency tables for all patients, detailing the comparison between imaging findings and motor results. Normal motor pathway findings on fMRI and DTI were found to significantly correlate with severe postoperative motor deficit after hemispherectomy.

ative motor decline. Of the 6 patients with no activation, 4 patients $(66.7 \%)$ remained stable, 1 patient $(16.7 \%)$ was mildly weaker, and 1 patient (16.7\%) was severely weaker postoperatively. Of the 13 patients with predominantly contralateral sensorimotor area activation on preoperative fMRI, $100 \%$ were severely weaker after surgery.

Among the 11 patients with perinatal stroke with fMRI activation, 5 patients $(45.5 \%)$ showed predominantly ipsilateral hemisphere activation, 3 patients $(27.3 \%)$ showed no activation at all, and 3 patients $(27.3 \%)$ showed contralateral activation. Postoperative motor function in 6 of 11 patients $(54.5 \%)$ with total perinatal stroke remained stable, 2 patients $(18.2 \%)$ were mildly weaker, and 3 patients $(27.3 \%)$ were severely weaker. Only the patients with contralateral fMRI activation preoperatively experienced severe postoperative motor decline.

The Freeman-Halton extension of the Fisher exact probability test was performed on the $3 \times 3$ contingency tables (Fig. 2) of all patients in our study. The 2-tailed p values, $\mathrm{p}_{\mathrm{A}}$ and $\mathrm{p}_{\mathrm{B}}\left(5.645 \times 10^{-5}\right)$, for fMRI were both statistically significant associations. We then condensed these data into $2 \times 2$ contingency tables to assess severe postoperative motor decline, including mildly weaker, stable, and improved postoperative motor examinations together as positive functional outcomes. Normal contralateral activation on motor fMRI predicted severe postoperative motor decline with $92.9 \%$ sensitivity (95\% CI 0.661-0.998) and $100 \%$ specificity $(95 \%$ CI $0.715-1.00)$.

On preoperative DTI, 13 of 25 patients (52.0\%) showed atrophic corticospinal tracts contralateral to the paretic limbs, and 12 patients (48.0\%) showed robust, essentially symmetric corticospinal tracts. Of the 13 patients with atrophic corticospinal tracts, 9 patients $(69.2 \%)$ remained stable, 2 patients $(15.4 \%)$ were mildly weaker postopera- tively, and 2 patients (15.4\%) were severely weaker after surgery. Of the 12 patients with symmetric, robust corticospinal tracts, $100 \%$ were severely weaker after surgery.

Among the 11 perinatal stroke patients with DTI, 9 patients $(81.8 \%)$ had atrophic corticospinal tracts contralateral to the paretic limbs, and 2 patients (18.2\%) showed symmetric, robust corticospinal tracts. On postoperative motor examination, 6 of 11 patients (54.5\%) with total perinatal stroke remained stable, 2 patients $(18.2 \%)$ were mildly weaker, and 3 patients $(27.3 \%)$ were severely weaker.

The Freeman-Halton extension of the Fisher exact probability test was performed on the $2 \times 3$ contingency tables (Fig. 2) of all patients who underwent DTI in our study. The 2-tailed $\mathrm{p}$ values, $\mathrm{p}_{\mathrm{A}}$ and $\mathrm{p}_{\mathrm{B}}$, for DTI were each 2.019 $\times 10^{-5}$, and both are statistically significant associations. Condensing these data into $2 \times 2$ contingency tables as above to assess severe postoperative motor decline, normal-appearing symmetric corticospinal tracts on DTI predicted severe postoperative motor decline with $85.7 \%$ sensitivity (95\% CI $0.572-0.982)$ and $100 \%$ specificity $(95 \%$ CI 0.715-1.00).

Preoperative hand motor function is associated with postoperative motor outcome $(p=0.012)$. However, when both fMRI and DTI findings agreed, outcome can be correctly predicted with $100 \%$ sensitivity and $100 \%$ specificity. In 11 patients, both fMRI and DTI findings were normal. Patients demonstrated severe postoperative hemiparesis in $100 \%$ of these cases. Conversely, in 11 patients, both fMRI and DTI findings were abnormal, and 100\% of these patients experienced, at worst, mild hemiparesis postoperatively. In 3 instances, the fMRI and DTI findings were in disagreement: fMRI correctly predicted outcome in 2 of these cases, and DTI correctly predicted outcome in 1 of these cases. 


\section{Interrater Reliability}

Thirteen reviewers independently scored the 25 available fMRI sensorimotor statistical parametric maps based on whether activation was absent, ipsilateral, or contralateral to the hemispherectomy. The $\kappa$ for interrater agreement was 0.69 , indicating good agreement $(\mathrm{p}<0.01)$. When asked to rate the CoFA-DTI maps as symmetric and robust on both sides or significantly atrophic on 1 side, the same 13 reviewers achieved $\kappa=0.64$, again indicating good agreement $(\mathrm{p}<0.01)$. When stratified by category, agreement was slightly higher for whether activation was ipsilateral or contralateral $(\kappa=0.71)$ compared with the presence or absence of activation $(\kappa=0.65)$. The survey results, as tabulated for interrater reliability evaluation, are available in Appendix B.

\section{Discussion}

Cerebral hemispherectomy has played a role in the treatment of epilepsy since the early 20 th century. ${ }^{20}$ Relative preservation of contralateral limb function, as well as increased mirror movements, has long been observed after cerebral hemispherectomy, and the implication is that the ipsilateral cortex provides motor function to the ipsilateral limb. Animal studies have demonstrated a critical maturation period in which the best outcomes are seen when the insults occur early in the postnatal period. ${ }^{38}$

In our study, normal motor fMRI predicted severe postoperative motor decline after functional hemispherectomy with excellent accuracy and good reliability $(92.9 \%$ sensitivity and $100 \%$ specificity), as did normal structural imaging of the corticospinal tracts using DTI $(85.7 \%$ sensitivity and $100 \%$ specificity). DTI analysis performed marginally better at predicting postoperative motor function loss. However, DTI was marginally less reliably interpreted among reviewers than fMRI. Our study illustrates the capacity of both advanced imaging techniques to reflect cortical reorganization.

\section{Ipsilateral Corticospinal Projections}

Ipsilateral signals are seen on electrocorticography during simple hand movement $\mathrm{s}^{32}$ and expanded in the setting of hemiplegia. ${ }^{21}$ In addition to contralateral projections, pyramidal pathways also contain a sizeable proportion of ipsilateral cortical projections, which appear to play a role in the control of distal extremity movement. Although most corticospinal fibers decussate in the medulla to the contralateral corticospinal tract, an estimated $8.2 \%$ of motor function is relayed through ipsilateral corticospinal fibers. ${ }^{1,36}$ Ipsilateral projections are suspected to play a role in the coordination of skilled movements in healthy subjects, possibly more so in the nondominant hand. The potential influence of the ipsilateral pathways is most robust in patients who have had a motor cortex stroke, and recent functional imaging studies support this concept of a subordinate collaborative motor pathway.

The recruitment of ipsilateral corticospinal neurons may promote coordination of fractionated movements. Hand movements seen after contralateral stroke are thought to occur due to projections to the red nucleus originating exclusively from the ipsilateral precentral gy- rus; these projections remain ipsilateral as they continue in the ipsilateral spinal corticospinal tract. ${ }^{2}$ Distal extremity movements, as well as axial body control, in stroke patients are frequently impaired compared with those of normal controls on the lesional side, though the contralateral corticospinal projections remain intact. ${ }^{3,10}$ Lesion data further support the role of ipsilateral projections in that, in patients undergoing cordotomy for cancer pain, the first unilateral lesion caused paresis of the ipsilateral lower limb with gradual recovery of limb function, whereas a second cordotomy on the contralateral side resulted in the immediate loss of all recovered motor function.

The dominant contralateral motor cortex likely regulates output from ipsilateral corticospinal neurons and, in a similar fashion, regulates brainstem pathways through upper motor neuron signaling at the level of the spinal cord. Müller et al. theorized that an increase in transcallosal inhibitory signaling could explain the finding that ipsilateral motor evoked potentials were absent after age 10 years, ${ }^{25}$ postulating that a contralateral motor cortex lesion might serve to unmask ipsilaterally mediated motor function. Ipsilateral sensorimotor cortex activation seen during paretic hand movements in stroke patients suggests that preexisting uncrossed neural pathways may be recruited to compensate for damage to the crossed motor pathways after ischemic stroke. ${ }^{3}$

\section{Predicting Motor Preservation After Hemispherectomy}

Several reports have demonstrated MRI evidence to suggest functional reorganization after cerebral hemispherectomy. ${ }^{11,12,14,15,40}$ Rutten et al. demonstrated activation in the undamaged hemisphere for both the paretic and normal hand preoperatively ${ }^{31}$ Postoperatively, fMRI was unchanged 22 months after hemispherectomy. De Bode et al. found that all children able to carry out the ankle dorsiflexion fMRI paradigm after functional hemispherectomy showed activations in the sensorimotor network ipsilateral to the affected side. ${ }^{5}$ Patients with perinatal infarct demonstrated greater activity in the cingulate cortex, whereas patients with Rasmussen encephalitis had significant activations in the insula, suggesting etiology-specific differences in reorganization.

The anatomical pathways by which this reorganization occurs are likely in place but are functionally or developmentally inhibited by contralateral hemisphere activity, while damaged pathways atrophy as shown on tractography (Fig. 3). Extensive damage could presumably lead to the loss of contralateral descending pyramidal connections and the loss of cross-hemispheric inhibition, mimicking the same state witnessed after surgical disconnection. This inhibition could partially explain the lack of preoperative ipsilateral fMRI activation in patients who retained baseline motor function after hemispherectomy.

Our patient demographics do suggest that a relatively young onset of cortical injury might increase the likelihood of motor reorganization. In addition, a longer period of time between injury and hemispherectomy might increase the likelihood of functional reorganization. To illustrate, of the 3 patients with hemimegalencephaly, 2 patients underwent hemispherectomy approximately 1 year after seizure onset, showed normal contralateral sensorimotor activation 


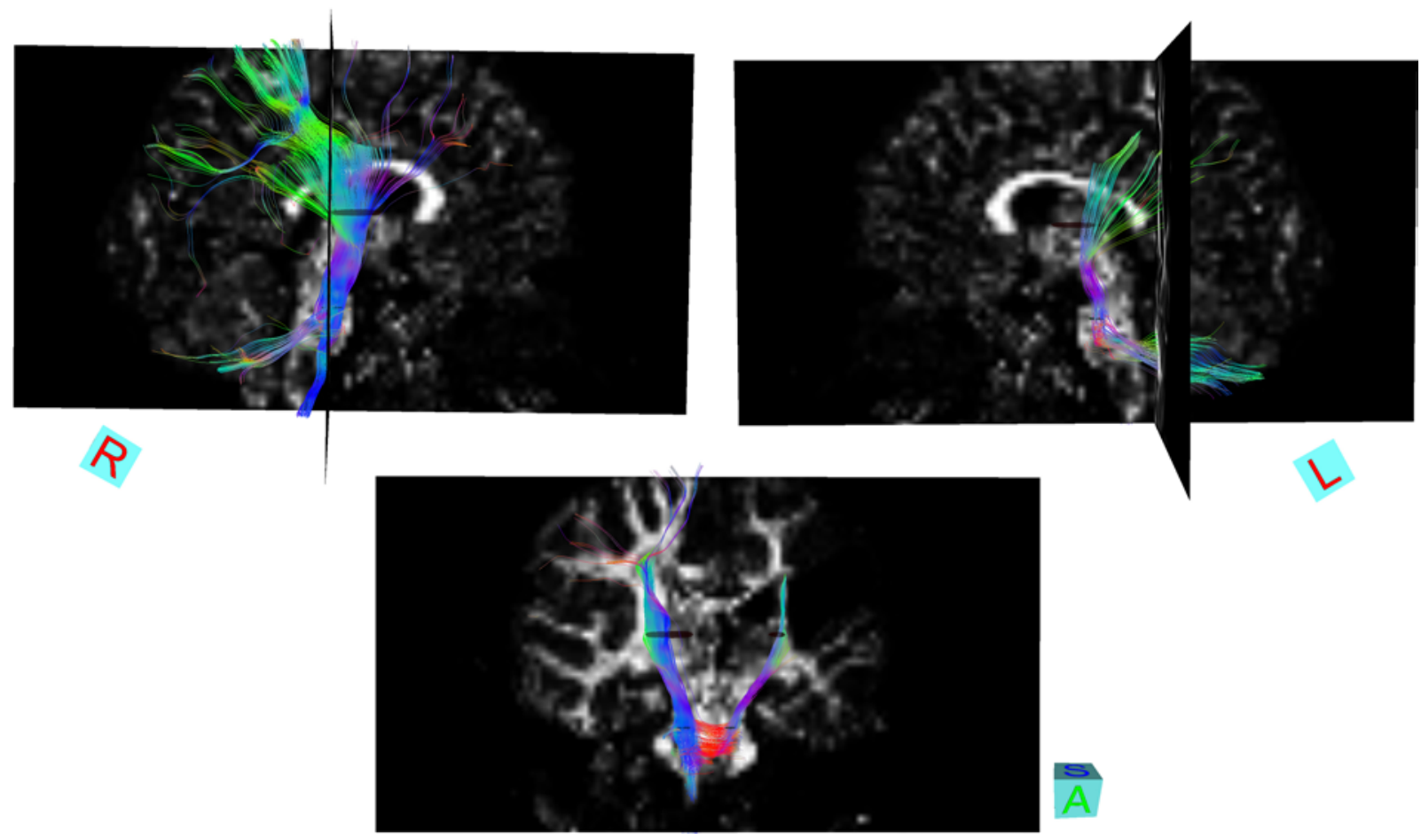

FIG. 3. DTI reconstructions. Standard clinical CoFA-DTI maps offer adequate information to assess corticospinal tract atrophy, which predicts motor outcomes postoperatively with good accuracy. These DTI reconstructions demonstrate a severely atrophic corticospinal tract contralateral to the paretic limb compared with a robust normal corticospinal tract on the ipsilateral side.

on fMRI with symmetric corticospinal tract integrity, and had severe motor dysfunction postoperatively. In contrast, in the third patient, approximately 6 years elapsed between seizure onset and hemispherectomy and the patient showed ipsilateral sensorimotor activation on fMRI and atrophy of the corresponding corticospinal tract and retained stable motor function postoperatively.

Patients with perinatal stroke, in particular, are likely to retain baseline motor function after hemispherectomy. In these patients, the functional results are in keeping with the "early lesion effect." 17 A DTI study by van der Aa et al. showed that fractional anisotropy values, while not initially lower after injury, do reflect functional pathway disruption at 3 months after perinatal stroke, and this alteration predicts cortical dysfunction in motor and visual pathways. ${ }^{37}$ Our data seem to support this supposition, in that only 3 of 11 stroke patients showed normal contralateral sensorimotor activation on $\mathrm{fMRI}$ - the same 3 perinatal stroke patients with severe motor decline postoperatively.

It remains unclear if neuronal pruning negates the ability to reorganize at a certain age or stage of development. However, the impressive ability to improve motor function postoperatively with aggressive rehabilitation, even years after surgery, is linked with sensorimotor fMRI activation, thereby suggesting recruitment of the ipsilateral corticospinal tracts. ${ }^{6}$ This finding has important implications regarding the potential value of preoperative motor conditioning leading up to functional hemispherectomy.

Our study has a number of limitations, thereby leaving several interesting questions unanswered. Its retrospective nature does not allow for a granular or uniform compari- son of pre- and postoperative motor function. In particular, a more detailed quantitative comparison of distal versus proximal extremity function would serve to elucidate the rubrospinal contribution relative to the ipsilateral corticospinal contribution, in that ipsilateral corticospinal control would be expected to manifest as relatively dysfunctional distal mobility. However, in terms of the SMA contribution, we observed 3 instances of contralateral SMA fMRI activation-once each in association with contralateral sensorimotor, ipsilateral sensorimotor-adjacent, and absent sensorimotor activation. Postoperative motor function was stable in the patients with ipsilateral and absent sensorimotor activation and severely weaker in patients with normal contralateral activation. This observation suggests, but certainly does not prove, that SMA is unlikely to be the major contributor to motor preservation.

The interpretation and generalizability of fMRI in this patient population remains ambiguous and potentially prone to motion and other artifacts, though our motor testing paradigm has proven reproducible. ${ }^{34}$ The fact that many patients were cognitively impaired adds to the risk that our fMRI results may be partially obscured by motion artifacts. The DTI protocol we routinely use in the clinical setting is basic, utilizing at first 10 and then later 30 directions that are repeated 2-4 times. Using a longer protocol with better resolution and more diffusion directions would yield better image and data resolution, though whether such improvement would yield any demonstrable benefit is uncertain. The small number of patients analyzed does not prove the adequacy of either imaging modality for predicting motor preservation, and a greater number of patients 
with multiinstitutional validation would serve to confirm the reliability of our findings, as well as the utility of these imaging modalities for this purpose.

\section{Conclusions}

Corticospinal tract atrophy on CoFA-DTI maps and abnormal sensorimotor activation on fMRI both demonstrate an excellent capacity for predicting severe motor decline after functional hemispherectomy in our series. Interpretation of these imaging techniques can be performed by subspecialists in multiple related disciplines with good agreement. When these imaging techniques were both in agreement, motor outcome was predicted correctly $100 \%$ of the time. These imaging techniques are useful for counseling about one of the most impactful patient-specific risks of undergoing functional hemispherectomy-hemiparesis.

It is clear from our patients and others that areas outside of the primary motor cortex can facilitate movement, excluding fractionated finger control. Release of cross-hemispheric inhibition likely promotes increased activation of the ipsilateral cortex. Erroneous, mistimed, or uncoordinated signaling from the damaged hemisphere could serve to inhibit intact ipsilateral motor control centers. Surgical disconnection seems to release this negative inhibitory influence, allowing ipsilateral motor centers to function unimpeded.

The mechanisms of reorganization seen in animal lesion models and patients who have had a stroke appear to provide a plausible explanation for the motor preservation seen in patients who undergo hemispherectomy, particularly those who have had a perinatal stroke. By understanding the mechanisms by which ipsilateral motor areas control isolateral limb function, strategies might be developed to use these native pathways with the hope of promoting neuroplasticity. One simple, potentially impactful strategy might be to promote aggressive motor training prior to functional hemispherectomy.

\section{References}

1. Benecke R, Meyer BU, Freund HJ: Reorganisation of descending motor pathways in patients after hemispherectomy and severe hemispheric lesions demonstrated by magnetic brain stimulation. Exp Brain Res 83:419-426, 1991

2. Brinkman J, Kuypers HG: Cerebral control of contralateral and ipsilateral arm, hand and finger movements in the splitbrain rhesus monkey. Brain 96:653-674, 1973

3. Cao Y, D'Olhaberriague L, Vikingstad EM, Levine SR, Welch KM: Pilot study of functional MRI to assess cerebral activation of motor function after poststroke hemiparesis. Stroke 29:112-122, 1998

4. Choi JT, Vining EP, Mori S, Bastian AJ: Sensorimotor function and sensorimotor tracts after hemispherectomy. Neuropsychologia 48:1192-1199, 2010

5. de Bode S, Firestine A, Mathern GW, Dobkin B: Residual motor control and cortical representations of function following hemispherectomy: effects of etiology. J Child Neurol 20:64-75, 2005

6. de Bode S, Mathern GW, Bookheimer S, Dobkin B: Locomotor training remodels fMRI sensorimotor cortical activations in children after cerebral hemispherectomy. Neurorehabil Neural Repair 21:497-508, 2007
7. Delalande O, Bulteau C, Dellatolas G, Fohlen M, Jalin C, Buret V, et al: Vertical parasagittal hemispherotomy: surgical procedures and clinical long-term outcomes in a population of 83 children. Neurosurgery 60 (2 Suppl):ONS19-ONS32, 2007

8. Devlin AM, Cross JH, Harkness W, Chong WK, Harding B, Vargha-Khadem F, et al: Clinical outcomes of hemispherectomy for epilepsy in childhood and adolescence. Brain 126:556-566, 2003

9. Fleiss JL: Measuring nominal scale agreement among many raters. Psychol Bull 76:378-382, 1971

10. Gazzaniga MS, Bogen JE, Sperry RW: Dyspraxia following division of the cerebral commissures. Arch Neurol 16:606612,1967

11. Govindan RM, Brescoll J, Chugani HT: Cerebellar pathway changes following cerebral hemispherectomy. J Child Neurol 28:1548-1554, 2013

12. Graveline CJ, Mikulis DJ, Crawley AP, Hwang PA: Regionalized sensorimotor plasticity after hemispherectomy fMRI evaluation. Pediatr Neurol 19:337-342, 1998

13. Hallett M: Plasticity of the human motor cortex and recovery from stroke. Brain Res Brain Res Rev 36:169-174, 2001

14. Holloway V, Gadian DG, Vargha-Khadem F, Porter DA, Boyd SG, Connelly A: The reorganization of sensorimotor function in children after hemispherectomy. A functional MRI and somatosensory evoked potential study. Brain 123:2432-2444, 2000

15. Ibrahim GM, Morgan BR, Smith ML, Kerr E, Donner E, Go CY, et al: Thalamocortical connectivity is enhanced following functional hemispherotomy for intractable lateralized epilepsy. Epilepsy Behav 51:281-285, 2015

16. Jonas R, Nguyen S, Hu B, Asarnow RF, LoPresti C, Curtiss $S$, et al: Cerebral hemispherectomy: hospital course, seizure, developmental, language, and motor outcomes. Neurology 62:1712-1721, 2004

17. Kennard MA: Age and other factors in motor recovery from precentral lesions in monkeys. Am J Physiol 115:138-146, 1936

18. Kossoff EH, Vining EP, Pillas DJ, Pyzik PL, Avellino AM, Carson BS, et al: Hemispherectomy for intractable unihemispheric epilepsy etiology vs outcome. Neurology 61:887890, 2003

19. Meoded A, Faria AV, Hartman AL, Jallo GI, Mori S, Johnston $\mathrm{MV}$, et al: Cerebral reorganization after hemispherectomy: a DTI study. AJNR Am J Neuroradiol 37:924-931, 2016

20. Miller JW, Silbergeld DL (eds): Epilepsy Surgery: Principles and Controversies. New York: Taylor \& Francis, 2006

21. Miller KJ, Abel TJ, Hebb AO, Ojemann JG: Reorganization of large-scale physiology in hand motor cortex following hemispheric stroke. Neurology 76:927-929, 2011

22. Moosa AN, Gupta A, Jehi L, Marashly A, Cosmo G, Lachhwani D, et al: Longitudinal seizure outcome and prognostic predictors after hemispherectomy in 170 children. Neurology 80:253-260, 2013

23. Moosa AN, Jehi L, Marashly A, Cosmo G, Lachhwani D, Wyllie E, et al: Long-term functional outcomes and their predictors after hemispherectomy in 115 children. Epilepsia 54:1771-1779, 2013

24. Mori H, Aoki S, Abe O, Hayashi N, Masutani Y, Ohtomo K, et al: Diffusion property following functional hemispherectomy in hemimegalencephaly. Acta Radiol 45:778-781, 2004

25. Müller K, Kass-Iliyya F, Reitz M: Ontogeny of ipsilateral corticospinal projections: a developmental study with transcranial magnetic stimulation. Ann Neurol 42:705-711, 1997

26. Nelles M, Urbach H, Sassen R, Schöne-Bake JC, Tschampa $\mathrm{H}$, Träber F, et al: Functional hemispherectomy: postoperative motor state and correlation to preoperative DTI. Neuroradiology 57:1093-1102, 2015 
27. Pascual-Leone A, Cohen LG, Brasil-Neto JP, Valls-Solé J, Hallett M: Differentiation of sensorimotor neuronal structures responsible for induction of motor evoked potentials, attenuation in detection of somatosensory stimuli, and induction of sensation of movement by mapping of optimal current directions. Electroencephalogr Clin Neurophysiol 93:230-236, 1994

28. Pascual-Leone A, Peris M, Tormos JM, Pascual AP, Catalá MD: Reorganization of human cortical motor output maps following traumatic forearm amputation. Neuroreport 7:2068-2070, 1996

29. Pascual-Leone A, Tarazona F, Catalá MD: Applications of transcranial magnetic stimulation in studies on motor learning. Electroencephalogr Clin Neurophysiol Suppl 51:157161, 1999

30. Pascual-Leone A, Walsh V, Rothwell J: Transcranial magnetic stimulation in cognitive neuroscience-virtual lesion, chronometry, and functional connectivity. Curr Opin Neurobiol 10:232-237, 2000

31. Rutten GJ, Ramsey NF, van Rijen PC, Franssen H, van Veelen CW: Interhemispheric reorganization of motor hand function to the primary motor cortex predicted with functional magnetic resonance imaging and transcranial magnetic stimulation. J Child Neurol 17:292-297, 2002

32. Scherer R, Zanos SP, Miller KJ, Rao RP, Ojemann JG: Classification of contralateral and ipsilateral finger movements for electrocorticographic brain-computer interfaces. Neurosurg Focus 27(1):E12, 2009

33. Schramm J, Behrens E, Entzian W: Hemispherical deafferentation: an alternative to functional hemispherectomy. Neurosurgery 36:509-516, 1995

34. Shurtleff H, Warner M, Poliakov A, Bournival B, Shaw DW, Ishak $\mathrm{G}$, et al: Functional magnetic resonance imaging for presurgical evaluation of very young pediatric patients with epilepsy. J Neurosurg Pediatr 5:500-506, 2010

35. Staudt M, Pieper T, Grodd W, Winkler P, Holthausen H, Krägeloh-Mann I: Functional MRI in a 6-year-old boy with unilateral cortical malformation: concordant representation of both hands in the unaffected hemisphere. Neuropediatrics 32:159-161, 2001

36. Tanji J, Kurata K: Contrasting neuronal activity in the ipsilateral and contralateral supplementary motor areas in relation to a movement of monkey's distal hindlimb. Brain Res 222:155-158, 1981

37. van der Aa NE, Northington FJ, Stone BS, Groenendaal F, Benders MJ, Porro G, et al: Quantification of white matter injury following neonatal stroke with serial DTI. Pediatr Res 73:756-762, 2013

38. Villablanca JR, Hovda DA: Developmental neuroplasticity in a model of cerebral hemispherectomy and stroke. Neuroscience 95:625-637, 2000
39. Zhang J, Mei S, Liu Q, Liu W, Chen H, Xia H, et al: fMRI and DTI assessment of patients undergoing radical epilepsy surgery. Epilepsy Res 104:253-263, 2013

40. Zsoter A, Pieper T, Kudernatsch M, Staudt M: Predicting hand function after hemispherotomy: TMS versus fMRI in hemispheric polymicrogyria. Epilepsia 53:e98-e101, 2012

\section{Disclosures}

The authors report the following. Dr. Ojemann is co-chair of the editorial board of JNS: Pediatrics and supported by the Richard G. Ellenbogen Endowed Chair of Neurological Surgery at Seattle Children's Hospital. Dr. Mathern is co-editor in chief of Epilepsia and Epilepsia-Open and on the editorial board of Neurology; he is supported by the Davies Endowed Chair in Honor of Paul H. Crandall for Epilepsy Research at the University of California, Los Angeles and the National Institutes of Health.

\section{Author Contributions}

Conception and design: Ojemann, AC Wang, Poliakov, Shurtleff, Warner. Acquisition of data: AC Wang, Ibrahim, Poliakov, PI Wang, Fallah, Buckley, Collins, Weil, Shurtleff, Warner, Perez, Shaw, Wright, Saneto, Novotny, Lee, Browd. Analysis and interpretation of data: Ojemann, AC Wang, Ibrahim, Poliakov, PI Wang, Fallah, Buckley, Collins, Weil, Perez, Shaw, Wright, Saneto, Novotny, Lee, Browd. Drafting the article: Ojemann, AC Wang, Ibrahim, Poliakov, PI Wang, Buckley, Weil, Browd. Critically revising the article: Ojemann, AC Wang, Ibrahim, Poliakov, Mathern, Saneto. Reviewed submitted version of manuscript: Ojemann, AC Wang, Ibrahim, Poliakov, PI Wang, Fallah, Mathern, Buckley, Collins, Weil, Shurtleff, Warner, Perez, Shaw, Wright, Saneto, Novotny. Approved the final version of the manuscript on behalf of all authors: Ojemann. Statistical analysis: AC Wang, Ibrahim, Fallah, Weil. Administrative/technical/material support: AC Wang. Study supervision: Ojemann, AC Wang.

\section{Supplemental Information}

\section{Online-Only Content}

Supplemental material is available with the online version of the article.

Appendices A and B. https://thejns.org/doi/suppl/10.3171/2017. 7.PEDS17137.

\section{Correspondence}

Jeffrey G. Ojemann, Seattle Children's Hospital, Department of Neurological Surgery, 4800 Sand Point Way NE, Seattle, WA 98105. email: jeff.ojemann@seattlechildrens.org. 